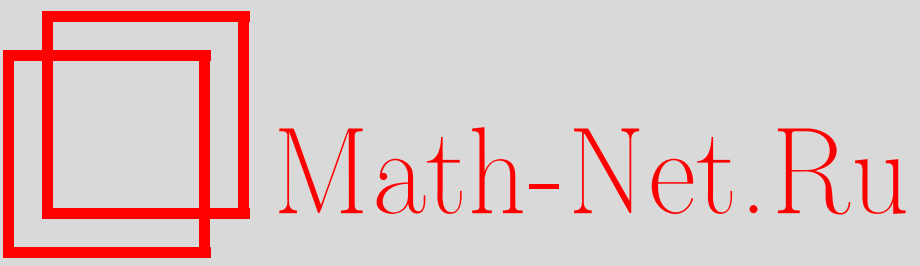

О. Е. Антоненкова, Ф. А. Шамоян, Описание линейных непрерывных функционалов в весовых пространствах аналитических в шаре функций со смешанной нормой, УМH, 2005, том 60, выпуск 4, 217-218

DOI: https://doi.org/10.4213/rm1451

Использование Общероссийского математического портала Math-Net.Ru подразумевает, что вы прочитали и согласны с пользовательским соглашением

http://www.mathnet.ru/rus/agreement

Параметры загрузки:

IP : 54.92 .164 .108

26 апреля 2023 г., 16:19:20 


\title{
ОПИСАНИЕ ЛИНЕЙНЫХ НЕПРЕРЫВНЫХ ФУНКЦИОНАЛОВ \\ В ВЕСОВЫХ ПРОСТРАНСТВАХ АНАЛИТИЧЕСКИХ В ШАРЕ ФУНКЦИЙ СО СМЕШАННОЙ НОРМОЙ
}

\author{
О. Е. АнтоненковА, Ф. А. ШАмоян
}

Пусть $B_{n}$ - открытьй единичньй шар в $n$-мерном комплексном пространстве, $S_{n}$ - его граница, $0<p, q<+\infty$. Обозначим через $\Omega$ множество всех положительных функций $\omega$, суммируемых на $(0,1)$, для которых существуют положительные числа $m_{\omega}, M_{\omega}, q_{\omega}$, причем $m_{\omega}, q_{\omega} \in(0,1)$, такие, что $m_{\omega} \leqslant \frac{\omega(\lambda r)}{\omega(r)} \leqslant M_{\omega}, \forall r \in(0,1), \lambda \in\left[q_{\omega}, 1\right]$. Важным частным случаем таких функций являются функции вида $\omega(t)=t^{\alpha}, \alpha>-1$. Свойства функций из класса $\Omega$ хорошо изучены в монографии [1]. Обозначим через $L^{p, q}(\omega)$ пространство измеримых в $B_{n}$ функций $f$, для котоpыx

$$
\|f\|_{L^{p, q}(\omega)}=\left(\int_{0}^{1} \omega(1-r)\left(\int_{S_{n}}|f(r \xi)|^{p} d \sigma(\xi)\right)^{\frac{q}{p}} r^{2 n-1} d r\right)^{\frac{1}{q}}<+\infty,
$$

а через $H\left(B_{n}\right)$ - множество всех голоморфных в $B_{n}$ функций. Положим также $A^{p, q}(\omega)=$ $H\left(B_{n}\right) \cap L^{p, q}(\omega)$. Подпространство пространства $L^{p, q}(\omega)$, состоящее из $n$-гармонических функций, обозначим через $h^{p, q}(\omega)$. В статье [2] получено описание линейных непрерывных функционалов в пространствах $A^{p, q}(\omega)$ в следующем частном случае: $\omega(t)=t^{\beta}, \beta>-1$, $1<p<+\infty, \max \{1,1+\beta\}<q<+\infty$. При остальных $p$ и $q$ метод, предложенньй в этой работе, не проходит. В этой заметке мы предлагаем другой подход, позволяющий описать линейные непрерывные функционалы в пространствах $A^{p, q}(\omega)$ при всех $0<p, q<+\infty$ и $\omega \in \Omega$. Для изложения этих резултатов введем обозначения. Пусть $\omega_{\alpha}(t)=\omega(t) \cdot\left(\frac{t^{\alpha}}{\omega(t)}\right)^{q}$, $t \in(0,1), \alpha_{\omega}=\frac{\log m_{\omega}}{\log q_{\omega}}, I=(0,1], J=(1,+\infty), D^{\alpha}$ - интегро-дифференциальный оператор Римана-Лиувилля дробного порядка $\alpha \in \mathbb{R}$. Для $\xi, z \in B_{n}$ положим $e_{z}=\frac{1}{1-\langle\xi, \bar{z}\rangle}$. Справедливо следующее утверждение.

Tеорема 1. Пусть $\Phi$ - линейный непрерывный функционал на $A^{p, q}(\omega), p, q \in J, u$ $g(z)=\Phi\left(e_{z}\right), z \in B_{n}$. Тогда $g \in H\left(B_{n}\right) u D^{\alpha+1} g \in A^{p^{\prime}, q^{\prime}}\left(\omega_{\alpha}\right), \alpha>\alpha \omega, p^{\prime}=\frac{p}{p-1}$, $q^{\prime}=\frac{q}{q-1}, \omega_{\alpha}(t)=\omega(t)\left(\frac{t^{\alpha}}{\omega(t)}\right)^{q^{\prime}}, t \in(0,1)$. Функционал

$$
\Phi(f)=\lim _{\rho \rightarrow 1-0} \int_{S_{n}} f(\rho \xi) \overline{g(\rho \xi)} d \sigma(\xi)
$$

при этом существуют положительные константы $c_{1}, c_{2}$ такие, что

$$
c_{1}\left\|D^{\alpha+1} g\right\|_{A^{p^{\prime}, q^{\prime}}\left(\omega_{\alpha}\right)} \leqslant\|\Phi\| \leqslant c_{2}\left\|D^{\alpha+1} g\right\|_{A^{p^{\prime}, q^{\prime}}\left(\omega_{\alpha}\right)} .
$$

Верно и обратное: любая функция $g$ такая, что $D^{\alpha+1} g \in A^{p^{\prime}, q^{\prime}}\left(\omega_{\alpha}\right)$, по формуле (1) порождает линейный непрерьвный функционал на $A^{p, q}(\omega)$, для которого справедливь оценки (2).

Чтобы сформулировать аналог этой теоремы для остальных $p, q$, введем следующие обозначения. Пусть $p, q \in I$. Обозначим через $\lambda^{p, q}(\omega)$ пространство функций $g \in H\left(B_{n}\right)$, для которых

$$
\|g\|_{\lambda^{p, q}(\omega)}=\sup _{z \in B_{n}}\left\{\frac{\left(1-|z|^{2}\right)^{\alpha-\frac{1}{q}+1-n\left(\frac{1}{p}-1\right)}}{\omega(1-|z|)^{1 / q}}\left|D^{\alpha+1} g(z)\right|\right\}<+\infty,
$$

где $\alpha>\frac{\alpha_{\omega}+1}{q}+n\left(\frac{1}{p}-1\right)-1$. Пусть далее $p \in J, q \in I$, обозначим через $\widehat{\lambda}^{p, q}(\omega)$ множество $g \in H\left(B_{n}\right)$, для которых

$$
\|g\|_{\widehat{\lambda}^{p, q}(\omega)}=\sup _{r \in(0,1)}\left\{\frac{(1-r)^{\alpha-\frac{1}{q}+1}}{\omega(1-r)^{1 / q}}\left(\int_{S_{n}}\left|D^{\alpha+1} g(r \xi)\right|^{p^{\prime}} d \sigma(\xi)\right)^{\frac{1}{p^{\prime}}}\right\}<+\infty,
$$


где $\alpha>\frac{\alpha_{\omega}+1}{q}-2$. Здесь $\frac{1}{p}+\frac{1}{p^{\prime}}=1$. Если $p \in I, q \in J$, то через $\widetilde{\lambda}_{\omega}^{p, q}$ обозначим пространство $g \in H\left(B_{n}\right)$, для которых

$$
\|g\|_{\widetilde{\lambda}^{p, q}(\omega)}=\left(\int_{0}^{1} \frac{(1-r)^{\alpha q^{\prime}-n q^{\prime}\left(\frac{1}{p}-1\right)}}{\omega(1-r)^{q^{\prime} / q}} \sup _{\xi \in S_{n}}\left|D^{\alpha+1} g(r \xi)\right|^{q^{\prime}} r^{2 n-1} d r\right)^{\frac{1}{q^{\prime}}}<+\infty,
$$

где $\alpha>\frac{\alpha_{\omega}}{q}+n\left(\frac{1}{p}-1\right)-\frac{1}{q^{\prime}}$. Здесь $\frac{1}{q}+\frac{1}{q^{\prime}}=1$. Можно установить, что определение этих классов не зависит от $\alpha$. Пусть пространство $\Lambda^{p, q}(\omega)$, где $p, q \in I \cup J$, совпадает с пространством $\lambda^{p, q}(\omega)$, если $p, q \in I$, с пространством $\widehat{\lambda}^{p, q}(\omega)$, если $p \in J, q \in I$, и с пространством $\widetilde{\lambda}^{p, q}(\omega)$, если $p \in I$, $q \in J$.

Tеорема 2. Пусть $p, q \in I \cup J, 0<\min \{p, q\} \leqslant 1$. Тогда если $\Phi-$ линейный непрерывньй функционал на $A^{p, q}(\omega)$ и $g(z)=\Phi\left(e_{z}\right), z \in B_{n}$, mo $g \in \Lambda^{p, q}(\omega)$ и $\Phi$ представим $в$ виде

$$
\Phi(f)=\lim _{\rho \rightarrow 1-0} \int_{S_{n}} f(\rho \xi) \overline{g(\rho \xi)} d \sigma(\xi)
$$

при этом существуют полохстельные константы $c_{1}, c_{2}$ такие, что

$$
c_{1}\|g\|_{\Lambda^{p, q}(\omega)} \leqslant\|\Phi\| \leqslant c_{2}\|g\|_{\Lambda^{p, q}(\omega)} .
$$

Верно и обратное: любая функция $g \in \Lambda^{p, q}(\omega)$ по формуле (3) порождает линейный непрерывный функционал на $A^{p, q}(\omega)$, для которого справедливы оценки (4).

Доказательство теорем 1 и 2 основано на следующих двух вспомогательных утверждениях, имеющих, на наш взгляд, самостоятельньй интерес. Для формулировки этих утверждений введем также следующие обозначения. Пусть $\alpha>-1, K_{\alpha}(z, \xi)=\frac{\omega(1-|\xi|)}{(1-\langle z, \xi\rangle)^{\alpha+n+1}}$, где $z, \xi \in B_{n}$, $T_{\alpha}(f)(z)=c(\alpha) \int_{B_{n}} K_{\alpha}(z, \xi) f(\xi) d \nu(\xi)$.

Лемма 1. Пусть $1 \leqslant p, q<+\infty, \alpha>\alpha_{\omega}$. Тогда оператор $T_{\alpha}$ отображает пространство $L^{p, q}(\omega)$ на пространство $A^{p, q}\left(\omega_{\alpha}\right)$, при этом справедлива оценка $\left\|T_{\alpha}(f)\right\|_{A^{p, q}\left(\omega_{\alpha}\right)} \leqslant c\|f\|_{L^{p, q}(\omega)}$.

Лемма 2. Предположим, что:

а) если $p, q \in I$, mo $\alpha>\frac{\alpha_{\omega}+1}{q}+n\left(\frac{1}{p}-1\right)-1$;

б) если $p \in I, q \in J$, mо $\alpha>\frac{\alpha_{\omega}}{q}+n\left(\frac{1}{p}-1\right)-\frac{1}{q^{\prime}}$, где $q^{\prime}=\frac{q}{q-1}$;

в) если $p \in J, q \in I$, mo $\alpha>\frac{\alpha_{\omega}+1}{q}-1$.

Тогда при всех наборах $p, q \in I \cup J, 0<\min \{p, q\} \leqslant 1$, оператор $T_{\alpha}(f)$ отображает пространство $h^{p, q}(\omega)$ на пространство $A^{p, q}\left(\omega_{\alpha}\right)$, причем $\left\|T_{\alpha}(f)\right\|_{A^{p, q}\left(\omega_{\alpha}\right)} \leqslant c\|f\|_{h^{p, q}(\omega)}$.

Аналоги этих результатов для других пространств голоморфных в поликруге функций получены в работах [3], [4]. Данные результаты имеют приложения в теории тёплицевых операторов.

При соответствующем выборе параметра $\alpha$ метод, используемый при доказательстве лемм 1 и 2 , позволяет нам построить в явном виде линейный ограниченный проектор из пространства $L^{p, q}(\omega)$ на соответствующее пространство $A^{p, q}(\omega)$ при $1 \leqslant p, q<+\infty$ и из пространства $h^{p, q}(\omega)$ на $A^{p, q}(\omega)$ при всех $p, q \in I \cup J$.

\section{СПИСОК ЛИТЕРАТУРЫ}

[1] Е. Сенета. Правильно меняющиеся функции. М.: Наука, 1985. [2] S. Gadbois // Proc. Amer. Math. Soc. 1988. V. 104. № 4. P. 1171-1180. [3] Ф. А. Шамоян // Сиб. матем. журн. 1990. Т. 31. № 2. С. 197-215. [4] Ф. А. Шамоян, О. В. Ярославцева // Записки научн. семин. ПОМИ. 1997. Т. 247. С. 268-275.

Брянский государственный университет E-mail: anto-olga@yandex.ru
Представлено В.М. Тихомировьпм Принято редколлегией 23.05.2005 